\title{
Editorial
}

\section{Evidence-based optimization of the fast-track approach to total knee replacement: do we need to interpret the information better?}

"Fast track" is the term used to describe a hospital admission in which the patient receives the best possible treatment based on the evidence and remains in hospital for no more than three days; moreover, the use of clinical resources is kept to the necessary minimum. The aim of the "fast-track" approach is to reduce perioperative morbidity and the length of the hospital stay (1).

The "fast-track" approach requires an informed and motivated patient; it also depends on logistical considerations related to the center where surgical treatment is carried out, and on the use of evidence-based treatment strategies across all areas of the patient's care, namely preoperative nursing, rehabilitation, anesthesia, pain control, reduction of bleeding and prevention of thromboembolic events, as well as management of any co-morbid conditions. There are numerous outcome measures that can be usefully considered to monitor the efficacy of "fast-track" treatment; of these, the length of hospital stay, the level of patient satisfaction, and the onset of complications can be considered the most important.

This issue of Joints includes two articles that, adopting a methodologically appropriate approach, look at the effectiveness of management of some intervention strategies related to the "fast-track" pathway in knee replacement surgery. Marra et al. (2) performed a prospective comparative study on the efficacy of the use of tranexamic acid in reducing blood loss after total knee arthroplasty (TKA), while Canata et al. (3), in a prospective randomized trial, compared different methods of postoperative pain management in patients undergoing TKA. There is no doubt that steps to reduce pain and blood loss after joint replacement surgery are crucial in ensuring a successful "fast-track" treatment, and there are many published studies that evaluate the effectiveness of both these and the other aspects of the surgery, mentioned above. The literature is full of evidence-based guidance on the best options to choose when managing a fast-track case, and the two new articles published in this issue of Joints will undoubtedly help to strengthen the current recommendations. However, a closer examination of published trials dealing with these topics reveals that although, overall, the treatment methods considered and analyzed are numerous, the studies themselves almost always confine themselves to comparing two techniques, and focus on just one aspect of the perioperative patient management (e.g. reducing bleeding, reducing pain, rehabilitation, and so on). Often, even systematic reviews with meta-analysis, taking into account the numerous direct paired comparisons that are furnished by the literature, struggle to go beyond mere estimation of the effect of one treatment compared with another. Consequently, traditional meta-analysis, being unable to extrapolate information on multiple comparisons (more than two treatments) and on combinations of treatments (e.g. the best combination of rehabilitation, prevention of bleeding, and pain management), fails to provide adequate interpretation of the available data. To overcome this, there exists a more sophisticated method of data analysis and interpretation, known as network meta-analysis (4, 5). Also called "mixed treatments comparison" or "multiple treatments comparison meta-analysis", this method broadens the scope of conventional paired meta-analysis by simultaneously analyzing direct comparisons between treatments within prospective randomized trials and indirect comparisons across trials based on a common "comparator" (e.g. indirect comparison of $A$ with $C$ through direct comparisons of $A$ with $B$ and of $B$ with $C$ ). It is a complex research method that requires a very cautious approach to the qualitative assessment of the trials included, as an error can be can greatly amplified during multiple indirect comparisons (6). Nevertheless, it may be an extremely powerful tool in the interpretation of the effectiveness of multiple and combined treatments, such as fast-track ones. And this must be recognized as an indispensable stage in the design of a fast-track treatment, especially considering the potential vulnerability of the population undergoing this type of surgery.

Giuseppe Milano Editor-in-Chief, Joints

\section{References}

1. Harsten A, Kehlet H, Toksvig-Larsen S. Recovery after total intravenous general anaesthesia or spinal anaesthesia for total knee arthroplasty: a randomized trial. Br J Anaesth. 2013;111:391-399.

2. Marra F, Rosso F, Bruzzone M, et al. Use of Tranexamic acid in total knee arthroplasty. Joints. 2016(4);202-213.
3. Canata GL, Casale V, Chiey A. Pain management in total knee arthroplasty: efficacy of a multimodal opiate-free protocol. Joints. 2016 (4);222-227.

4. Lumley T. Network meta-analysis for indirect treatment comparisons. Stat Med. 2002;21: 2313-2324.

5. Caldwell DM, Ades AE, Higgins JP. Simultaneous comparison of multiple treatments: combining direct and indirect evidence. $B M \mathrm{~J}$. 2005;331:897-900.

6. Li T, Puhan MA, Vedula SS, et al. Network meta-analysis-highly attractive but more methodological research is needed. BMC Med. 2011;9:79. 